\title{
Eye muscle potentiation does not account for adaptation in distance perception based on oculomotor cues
}

\author{
HANS WALLACH and PETER HALPERIN \\ Swarthmore College, Swarthmore, Pennsylvania 19081
}

\begin{abstract}
Ebenholtz and Wolfson have demonstrated an aftereffect of sustained ocular convergence, which they ascribed to eye muscle potentiation. They suggested that this effect can explain an aftereffect of wearing glasses that alter oculomotor cues for distance. Wallach and Frey interpreted this aftereffect as resulting from adaptation. The outcome of two experiments designed to test Ebenholtz and Wolfson's explanation and a review of previous experiments on adaptation in distance perception based on oculomotor cues show that this explanation is untenable.
\end{abstract}

Ebenholtz (1974) and Ebenholtz and Wolfson (1975) have demonstrated the existence of a perceptual aftereffect of sustained convergence. They ascribed this aftereffect to prolonged straining of the medial or lateral extraocular muscles, which results in a reflexive innervation of these muscles which lasts beyond the time of attempted relaxation. They called this aftereffect "eye muscle potentiation." It is assumed to result in a nonveridical ocular position signal and, inasmuch as convergence of the eyes serves as a distance cue, in nonveridical distance information. This aftereffect is induced by prolonged fixation at distances either shorter or farther than $29.4 \mathrm{~cm}$. The distance of $29.4 \mathrm{~cm}$ is the empirically determined point of balance between action of the medial and lateral recti muscles. "For example, if, as a result of maintained convergence on nearby objects, there was a continued (reflexive) innervation of the convergence mechanism, then in order to fixate on any given target the subject would have to overcome this reflexive innervation by some amount of voluntary innervation in the opposite direction, i.e., to diverge. This in turn would lead to the sense of an increase in distance to the fixated target" (Ebenholtz \& Wolfson, 1975, pp. 485-486).

The actual findings were that 6 -min fixation at a single distance nearer than $29.4 \mathrm{~cm}$ resulted in increased distance estimations, while fixation of a distance farther than $29.4 \mathrm{~cm}$ resulted in underestimation of distance. In both cases, the aftereffects increased with increasing deviation of the fixated distance from the balancing distance of $29.4 \mathrm{~cm}$. Fixating successively at different distances also

This work was supported by Girant BNS75-19095 A01 from the National Science Foundation Io Swarthmore Collegc, Hans Wallach, principal investigator. resulted in overestimation or underestimation of distance, depending upon whether the range of the fixation distances was nearer or farther than the balancing distance. Eight minutes of such exposure resulted in aftereffects roughly half as great as $6 \mathrm{~min}$ of static eye position.

Ebenholtz and Wolfson (1975) suggested that eye muscle potentiation (EMP) might account for the change in the evaluation of convergence that, according to Wallach and Frey $(1972 a, 1972 b)$ and Wallach, Frey, and Bode (1972), results from the adaptation to spectacles that alter these oculomotor cues. One pair of spectacles used by Wallach and his collaborators consisted of -1.5 -diopter spherical lenses and 5-diopter prisms, whose bases were in temporal position. These spectacles force a corresponding addition to whatever accommodation and convergence are required by the target distance and are referred to as "near glasses." The other spectacles consisted of +1.5-diopter spherical lenses and 5-diopter prisms, base nasal. They caused oculomotor adjustments to be decreased by the equivalent of 1.5 lens diopters, and are referred to as "far glasses" (Wallach \& Frey, 1972a, p. 77). A series of experiments indicated that wearing these glasses for $20 \mathrm{~min}$ under conditions where veridical distance cues were also present resulted in an adaptive recalibration of oculomotor cues. Thus, adaptation to near glasses resulted in increased registered distances, and wearing far glasses caused registered distance based on convergence and accommodations to become smaller.

Ebenholtz and Wolfson (1975) contended that wearing near glasses was equivalent to forcing convergence for distances shorter than $29.4 \mathrm{~cm}$ and wearing far glasses was equivalent to forcing fixation for distances beyond this point. Since this represents the condition under which EMP occurs, they say: "Given this account of distance aftereffects [by 
which they mean EMP], the questions must remain open as to whether recalibration does indeed occur at all (Ebenholtz \& Wolfson, 1975, p. 490).

The claim that wearing near glasses is equivalent to forcing convergence for distances shorter than $29.4 \mathrm{~cm}$ is, however, not correct. Only real distances shorter than $52.6 \mathrm{~cm}$ are viewed with convergence for distances of $29.4 \mathrm{~cm}$ or less when the near glasses are worn. ${ }^{1}$ Therefore, only when actual viewing distances during the exposure were consistently shorter than $52.6 \mathrm{~cm}$ could EMP possibly account for the results of the adaptation experiment. This was not the case in any of the adaptation conditions used by Wallach, Frey, and Bode (1972) where near glasses were used. In the description of their Experiment 5, for instance, where three specific viewing distances amounting to $33.3,66.7$, and $133.3 \mathrm{~cm}$ were employed, the authors stated that "the distances for which the eyes behind the glasses were adjusted were $22.2,33.3$, and $44.4 \mathrm{~cm}$ " (p. 115). Of these three convergence distances, two were larger than the balancing distance of $29.4 \mathrm{~cm}$ and one was shorter. This condition should be expected to yield either no EMP effect at all or one in the direction opposite to the obtained adaptation effect. In the adaptation condition that produced the strongest effect, the subject walked through a building while wearing the near glasses, and real viewing distances of less than $52.6 \mathrm{~cm}$ occurred seldom.

It seems thus very unlikely that eye muscle potentiation can account for the effects that Wallach, Frey, and Bode ascribe to adaptive recalibration of oculomotor cues. But with Ebenholtz's claim having appeared in reputable journals, we found it necessary to compare EMP and adaptation to near glasses in specifically designed experiments. Two experiments were performed. In Experiment 1, the effects of EMP and of adaptation to spectacles were measured under optimal conditions so that the magnitudes of the effects could be compared. In Experiment 2, subjects were exposed to two similar conditions. One was known to produce the adaptation effect and the other was such that no adaptation could develop. The two conditions were equally likely to produce the effect of eye muscle potentiation. They differed, in fact, only with regard to the presence or absence of veridical distance cues. Adaptation requires their presence and EMP does not. If the two conditions were found to yield aftereffects in different amounts, recalibration of distance cues must be assumed to occur.

Unpublished experiments by the senior author had shown that recalibration of distance cues is best measured with estimates of perceived size. This indirect method is based on the fact that perceived size varies in proportion to registered distance. Therefore, an increase in perceived size after the exposure period, when object size and observation distance remain unaltered, measures a proportional change in the relation between the affected distance cues and registered distances. Four out of the five experiments performed by Wallach, Frey, and Bode (1972) employed the size estimation test. ${ }^{2}$ Since our experiments were done to show that the results of the experiments by these authors were largely due to recalibration of distance cues, the use of this testing procedure was justified.

\section{EXPERIMENT 1}

\section{Method}

EMP was induced by having subjects view television on a 5 -in. set. Its screen was inserted in a hole in the center of a black cardboard, $60 \mathrm{~cm}$ wide and $60 \mathrm{~cm}$ high. The cardboard completely filled the subject's visual field, and thus insured that no light from the TV picture illuminated this part of his visual field. A small black cross was fixed to the center of the screen as a fixation point. The 1.5-diopter near glasses were employed for the adaptation condition.

The same tests were given in connection with EMP and the adaptation condition. The test object was similar to the larger of the two wire pyramids used by Wallach, Frey, and Bode (1972). It was constructed of $3.2-\mathrm{mm}$ rods painted black. Its base was $8 \mathrm{~cm}$ square. Two thin wires, $.6 \mathrm{~mm}$ thick, were stretched across it, bisecting opposite sides. They served as targets for accurate accommodation and convergence. The pyramid was mounted in a diamond-shaped aperture in the panel of a black box, with the base in the plane of the panel and the apex inside the box. The aperture was $12.7 \mathrm{~cm}$ and the pyramid base was in concentric position. A translucent sheet of white plastic material inside the box was dimly illuminated from the rear and served as the bright background against which the pyramid was visible. The box prevented the light that made the pyramid visible from illuminating the rest of the subject's field of vision. A biteboard was used to keep his head in a fixed position so that his eyes were $66.7 \mathrm{~cm}$ from the pyramid and on a level with it. This distance was selected because it was one of iwo test distances used by Wallach, Frey, and Bode. Built into the mounting of the biteboard was a microswitch that controlled the bulbs in the light box. By pushing the biteboard $1 \mathrm{~mm}$ forward, the subject could make the background of the pyramid light up. Hence, the pyramid was visible only when the subject's head was in the proper position, and only accommodation and convergence were available as cues for the pyramid's distance. The perceived size of the pyramid base was used as the measure for the registered distance that resulted from these oculomotor cues. When alteration of these distance cues due to adaptation or due to EMP increased registered distances, the pyramid base would be perceived larger than prior to exposure to one of these conditions. To measure this change in perceived size, subjects gave two estimates of the apparent length of one of the rods forming the base of the pyramid, one before and the other after the exposure. He did this by selecting and by adjusting the length of one of a series of thin metal rods without looking at them. ${ }^{3}$ Using the sense of touch only was required because changed visual size perception in the postexposure tests would have affected the apparent visual size of the test rods.

The two experimental conditions differed only with regard to the exposure procedure used. In the adaptation exposure, the subject wore the near glasses for $20 \mathrm{~min}$ and was taken on a tour of the science building by the experimenter, through long hallways and up and down stairs. In the EMP exposure, the subject was seated in front of the TV set with his head immobilized in a head- 
ress. The TV screen was adjusted for low brightness and placed at the subject's eye level. The subject was instructed to heep his eyes on the fixation matk throughout the exposure period, which also lasted $20 \mathrm{~min}$. The TV screen was $25 \mathrm{~cm}$ from the subjecl'? eyes. This distance was selected because it is the standard distance for near viewing by aduls. Our tesuls showed that is produced a good EMP effect. At the end of the EMP exposure period, the subject closed his eyes and was led by the experimenter to the tert appatatus and properly seated at the biteboard. The time intersal between the end of the viewing of the TV screen and the vart of the viewing of the test pyramid was always 1 min. The same standardized time interval separated the removal of the near glasses and the beginning of the test in the adaptation condition.

Subjects were selected on the basis of a pilot study which had yielded a mean and a standard deviation for the preexposure size estimate. Subjects whose preexposure size estimate differed from that mean by more than two standard deviations did not further participate in the experiment. Twelve undergraduates adapted to the near glasses and 15 were given the EMP exposure.

\section{Results}

The mean size estimates before and after the adaptation period are given in the first column $(1 \mathrm{~A})$ of Table 1. Adaptation to the near glasses caused an increase in perceived size of $45 \%$, a mean effect that approaches the one obtained by Wallach, Frey, and Bode (1972) in a similar experiment. The corresponding results obtained in connection with sustained convergence for $25 \mathrm{~cm}$ are given in column $1 \mathrm{~B}$. This EMP effect, a $15 \%$ change in perceived size was highly significant and agreed with the result obtained by Ebenholtz and Wolfson (1975) with a single target distance of $16.23 \mathrm{~cm}$ in their Experiment 2. The effect of adaptation was three times as large as the effect of sustained convergence, and the difference between the two mean effects was highly significant $(\mathrm{p}<.001)$.

\section{EXPERIMENT 2}

Our first experiment had measured adaptation to near glasses under optimal conditions and the aftereffect of sustained convergence for a distance that might conceivably occur during adaptation to near glasses. Besides providing data for a comparison of the magnitude of the effects, this experiment validated the size estimation test as appropriate for measuring the effect of EMP. But the two effects we compared were produced under conditions that in-

Table 1

Mean Size Estimates in Centimeters

\begin{tabular}{lcccc} 
& $1 \mathrm{~A}$ & $1 \mathrm{~B}$ & $2 \mathrm{~A}$ & $2 \mathrm{~B}$ \\
\cline { 2 - 5 } \multicolumn{1}{c}{$\begin{array}{c}\text { Exposure } \\
\text { Condition }\end{array}$} & $\begin{array}{c}\text { Adap- } \\
\text { tation }\end{array}$ & EMP & $\begin{array}{c}\text { Adap- } \\
\text { tation }\end{array}$ & EMP \\
\hline Preexposure & 7.6 & 8.13 & 8.01 & 8.29 \\
Postexposure & 11.03 & 9.36 & 10.23 & 8.38 \\
Difference & 3.43 & 1.23 & 2.22 & .09 \\
Proportional Change & $45 \%$ & $15 \%$ & $27.7 \%$ & $1.1 \%$ \\
\hline
\end{tabular}

Note-For $1 A, t(11)=10.3, p<.001 ;$ for $1 B, t(14)=4.77$, $p<.001 ;$ for $2 A, t(9)=5.0, p<.001 ;$ for $2 B, t(9)=1.0, p>.3$. volved very different ocular convergences. By contrast, Experiment 2 employed the same three discrete observation distances during the two different exposure condiiions. Subjects wore the near glasses during both exposures, causing nonveridical oculomotor adjustments that were, however, the same in the two exposure conditions. Therefore, if it were true that the adaptation condition produced its effect only because it caused an EMP effect, the two condilions should produce the same changes in registered distance and hence the same alteration in perceived size. The two conditions differed only in that the adaptation condition provided veridical distance cues, which were absent in the EMP condition.

\section{Method}

Three kinds of distance cues gave information about the true distances from the eyes of the three targets: perspective cues, the retinal image size of familiar objects, and head-movement parallax. Three $\$ 1$ bills mounted on black cardboard were the familiar objects and also provided the perspective cues, inasmuch as they caused identically shaped retinal images of different size. Throughout the exposure period, the subject turned his head from side to side and thereby obtained further cues for the true distances of the dollar bills from the eyes. Since the bills were never simultaneously visible, the parallactic cues they provided were for these absolute distances only. ${ }^{4}$

In the EMP condition, these distances cues were eliminated as follows. The dollar bills were replaced by luminous disks, circular apertures placed in front of light boxes with translucent front surfaces. A thin vertical wire was stretched across the center of each disk to serie as a sharp target for correct conıergence of the eyes. The sizes of the disks and the wires were so chosen that they were larger in proportion to their distances from the subject's eyes and produced retinal images of equal size. Each disk subtended a visual angle of $1.45 \mathrm{deg}$. The light boxes were mounted in such a way that the center of the disks were at subjecr's eye level. This arrangement took care of the cues of familiar size and of perspective. Head movements were eliminated by having the subject's head immobilized by a biteboard. The experimenter lit the disks one at a time for $5 \mathrm{sec}$ followed by a 1 -sec dark interval. A 12-position switch made it possible 10 randomize the order. The same lighting sequence was used in the adaptation exposure, where three concealed narrow-beam lamps illuminated each dollar bill separately. The proper distance of the subject's eyes from the bill was maintained by having the subject wear a welder's headgear that was attached to a vertical shaft which permitted the subject to turn his head.

The distances of the disks or of the dollar bills from the ubject's eyes were 50,200 , and $350 \mathrm{~cm}$. The near glasses, which were worn in both conditions, caused these targets to be viewed with accommodation and convergence for $28.6,50$, and $56 \mathrm{~cm}$. Both exposures lasted $20 \mathrm{~min}$. The tests were those of Experiment 1 and were administered in the same fashion. Ten subjects, selected in the same manner as before, were employed in the adaptation condition and another 10 in the EMP condition.

\section{Results}

Mean pre- and postadaptatoin size estimates are given in the third and fourth columns ( $2 \mathrm{~A}$ and $2 \mathrm{~B}$ ) of Table 1. Adaptation caused an increase in apparent size of $27.7 \%$. The EMP exposure had no effect; an increase in apparent size of $.09 \mathrm{~cm}$ was obtained with confidence limits at the .01 level amounting 11$) \pm .29 \mathrm{~cm}$. We can therefore conclude that, 
with a probability of .01 , a size increase of $1.84 \mathrm{~cm}$, i.e., of $2.22-(.09+.29)$, or of $23 \%$, could not have been due to EMP but was an adaptation effect.

\section{CONCLUSION}

This result of Experiment 2 was expected. There was not a single one among the five experiments reported by Wallach, Frey, and Bode (1972) where the convergence distance produced by the near glasses was consistently smaller than the balancing distance of $29.4 \mathrm{~cm}$. Since the real viewing distance that corresponds to a convergence distance of $29.4 \mathrm{~cm}$ when the near glasses are worn is $52.6 \mathrm{~cm}$, the preponderance of the convergence distances that occurred in the exposure periods in the experiments by Wallach, Frey, and Bode were larger rather than smaller than the balancing distance and, if an EMP effect were here to develop at all, it should have been opposite to that obtained by these authors. In Experiments 1 and 3 , where subjects walked through a college building, real viewing distances shorter than $52.6 \mathrm{~cm}$ occurred only rarely, when the subject approached a door that had to be opened or passed a person coming toward him. In Experiments 2 and 4, real viewing distances of less than $52.6 \mathrm{~cm}$ never occurred, and an EMP effect could only have diminished the adaptation effect. In Experiment 5, two of the three discrete convergence distances used were larger than the balancing distance. Had an EMP effect developed in any one of these experiments, it should have diminished the effect of adaptation.

The question may be raised as to how to deal with a possible EMP effect that may enhance or diminish an adaptation effect when, for some reason, exact measurements of the latter are needed. One answer is to use the scheme of our Experiment 2 and present in a control experiment the sequence of convergence distance that occurs in the adaptation exposure, but with veridical distance cues absent. It is, however, not at all sure that EMP develops when convergence changes continuously, particularly if muscle relaxation can occur because convergence for the balancing distance occurs from time to time. The EMP condition in our Experiment 2 yielded virtually no aftereffect. Had there been one, it should have caused a decrease in registered distance and therefore in perceived size rather than an increase as was produced by the adaptation condition, because, of the three convergence distances that occurred in the exposure period, two, that is, those of 50 and $56 \mathrm{~cm}$, were larger than the balancing distance and the third distance $(28.6 \mathrm{~cm})$ was near the balancing distance. Yet, no effect of decreased registered distance was obtained. It seems that convergence for the balancing distance during one-third of the exposure period prevented an EMP effect from accumulating. Whether even the presence of the balancing distance is necessary for avoiding an EMP effect from developing or whether continuous convergence changes suffice is, of course, an open question.

\section{REFERENCES}

Eвenholtz, S. M. The possible role of eye-muscle potentiation in several forms of prism adaptation. Perception, 1974, 3, 477-485.

Ebenholtz, S. M., \& Wolfson, D. M. Perceptual aftereffects of sustained convergence. Perception \& Psychophysics, 1975, 17. 485-491.

W AllaCh, H., \& Frey, K. J. Adaptation in distance perception based on oculomotor cues. Perception \& Psychophysics, 1972, 11, 31-34. (a)

Wallach, H., \& Frey, K. J. On counteradaptation. Perception \& Psychophysics, 1972, 11, 161-165. (b)

Wallach, H., Frey, K. J., \& Bode, K. A. The nature of adaptation in distance perception based on oculomotor cues. Perception \& Psychophysics. 1972, 11, 110-110.

W AllaCH, H..\& SMITH, A. Visual and proprioceptive adaptation to altered oculomotor adjustments. Perception \& Psychophysics, $1972,11,413-416$.

Wallach, H., Yablick, G. S., \& SMith, A. Target distance and adaptation in distance perception in the constancy of visual direction. Perception \& Psychophysics, 1972, 12, 139-145.

\section{NOTES}

1. Focusing at a distance of $52.6 \mathrm{~cm}$ involves an accommodation of the eves worth 1.9 lens diopters. With the near glasses forcing an additional accommodation of 1.5 diopters, aciommodation of the eyes amounts to 3.4 diopters when a point $52.6 \mathrm{~cm}$ distant is viewed through the near glasses. An accom. modation of 3.4 diopters, however, corresponds to a siewing distance of $29.4 \mathrm{~cm}$. Since the near glasses cause, for the average subject, a corresponding change in convergence, the eyes also are converged for a distance of $29.4 \mathrm{~cm}$ when the target distanci is 52.6 .

2. The size estimation test was also used successfully in some of the experiments by Wallach and Frey (1972a, 1972b), by Wallach and Smith (1972), and by Wallach, Yablick, and Smith (1972) to measure recalibration of oculomotor cues to distance.

3. For details of this estimation method, see Wallach and Frey (1972a), p. 79.

4. An unpublished experiment by Wallach and O'Leary has shown that such parallactic cues can serve as the sole veridical distance cues in adaptation to near glasses.

(Received for publication May 2, 1977; accepted July 22,1977 .) 